\title{
Dal packaging all'errore: casi esemplari e aspetti normativi
}

\author{
- Luciana Pazzagli ${ }^{1}$, Francesco Ranzani ${ }^{2}$, Elena Albini ${ }^{3}$ \\ 1 Farmacista. Centro di Farmacovigilanza, Azienda Sanitaria Firenze, Area Informazione e Technology Assessment. Società Italiana Farmacia \\ Ospedaliera \\ 2 Designer. Gestione Rischio Clinico e Sicurezza del paziente Regione Toscana. Società Italiana di Ergonomia \\ 3 Avvocato, Foro di Milano
}

\begin{abstract}
A number of factors can contribute to medication errors, and packaging may be one cause. For example a medication can be mistakenly administered either because the drug container (eg, iv bag, prefilled syringe, etc.) is similar in appearance to the intended medication's container or because the packages had similar labelling. The Authors describe three case reports that show how an unclear packaging can be responsible for inappropriate use, and subsequent patient's injuries. Hospital pharmacy staff, prescribing physicians, other healthcare workers, and even patients can play a role in minimizing the occurrence of these types of errors. However also drug manufacturers have an important role and should provide clear and unique labels and packages for their products, as underlined by pharmaceutical packaging legislation and regulations.
\end{abstract}

Keywords: packaging, labelling, medication error

From packaging to error: exemplary case reports and normative aspects

Pratica Medica \& Aspetti Legali 2009; 3(3): 131-137

\section{INTRODUZIONE}

Questo articolo desidera mettere in risalto i rischi che possono derivare dal confezionamento (packaging) di medicinali e prodotti salutistici, e quando questo può essere fonte di errore se non finalizzato alla prevenzione del rischio (il cosiddetto "packaging sicuro"). Si descrivono tre casi esemplificativi: tali casi, pur essendo riferiti a prodotti diversi dai farmaci, individuano però problematiche comuni anche al confezionamento di questi ultimi, tanto più che, nel verificarsi di eventi avversi, portano il paziente a ricorrere al medico, che necessita perciò di essere consapevole del rischio di errore indotto dal packaging. Larticolo desidera nel contempo fornire delle specifiche tecniche per diffondere la cultura della sicurezza; vengono infine sottolineati gli aspetti normativi relativi al confezionamento dei prodotti farmaceutici.

\section{IL RUOLO DEL PACKAGING}

Nel mondo dei prodotti farmaceutici, intendendo per tali non solo i farmaci ma tanti altri prodotti quali alimenti speciali, prodotti omeopatici, cosmetici e prodotti di derivazione naturale, il confezionamento ha una funzione multipla che va dal ruolo di "contenitore" a quello di "informatore" sulle caratteristiche tecniche. Il confezionamento è dunque un elemento di riferimento per l'acquisto (se prodotto in libera vendita) e per l'uso, sia da parte di cittadini/pazienti che di operatori sanitari.

Non sempre l'utilizzatore ricorre alle istruzioni di schede tecniche o foglietti illustrativi, per motivi che vanno dalla lettura frettolosa alla mancata comprensione di testi tecnici o all'inconscia sicurezza nella somministrazione/utilizzo: l'utilizzatore, ad esempio, si può basare sulle informazioni essenziali immediatamente rilevabili dalla confe- 
zione e/o fidarsi della "confidenza d'uso" acquisita nel tempo, senza pensare che ciò può essere fonte di errore e di conseguente rischio clinico.

L'impatto visivo, per leggibilità e chiarezza, dovrebbe in sintesi dare risalto alle informazioni essenziali per l'uso ed essere finalizzato alla sicurezza piuttosto che a strategie di marketing o logiche commerciali.

\section{SPECIFICHE TECNICHE}

Il confezionamento si distingue in due elementi fondamentali:

- confezionamento primario, ossia ciò che riveste direttamente il farmaco/prodotto: per esempio blister per compresse, fiala di vetro, flacone;

- confezionamento secondario, ossia ciò che riveste il confezionamento primario.

Entrambi questi elementi devono assolvere compiti fondamentali, quali proteggere il prodotto, assicurare l'assenza di interazioni negative tra contenitore e sostanza, renderne facile e funzionale l'uso, ma, al contempo, impedire la manomissione o gli usi impropri (per esempio da parte dei bambini), garantire l'immediata identificazione visiva del farmaco/prodotto e del suo dosaggio, ma anche impedire scambi dovuti a imballaggi o grafica non chiari.

La peculiarità del prodotto (farmaco, integratore, ecc.) obbliga al rispetto di norme (buona preparazione, autorizzazione al commercio, ecc.) e il design del packaging dovrebbe includere un insieme di indicazioni obbligatorie, buon senso e progettazione al servizio degli utilizzatori. Tuttavia raramente per la scelta del confezionamento si effettuano studi di progettazione basati su User Centered Design.

Il Centro Gestione Rischio Clinico e sicurezza del paziente della Regione Toscana (GRC), SIFO Toscana (Società Italiana di Farmacia Ospedaliera e dei Servizi Farmaceutici delle ASL) e SIE (Società Italiana di Ergonomia) hanno divulgato una Carta di raccomandazioni per il "packaging sicuro" per implementare lo sviluppo di una specifica cultura e promuovere azioni verso l'Agenzia Italiana del Farmaco e Farmindustria (vedi box nella pagina seguente per maggiori dettagli).

\section{CASI ESEMPLARI}

\section{ALIMENTO SPECIALE: PRODOTTO E TESTO POCO VISIBILI}

Questo primo caso riguarda una paziente sottoposta a nutrizione enterale. La sacca contenente il liquido per la nutrizione enterale era stata attaccata al deflussore e la pompa era stata avviata; l'alimento però non era in grado di defluire, in quanto coagulato, generando così un errore nella pompa, con un conseguente segnale acustico che aveva allertato i familiari. Nel frattempo una piccola quantità di liquido e aria, passati in sonda, avevano prodotto un lieve malore nella paziente. Il buon funzionamento della pompa per nutrizione enterale ha permesso tuttavia di comprendere e risolvere il problema.

Ma come mai gli operatori sanitari non si erano accorti che il liquido per la nutrizione enterale fosse coagulato? La causa è da ricercare nel packaging del prodotto.

Il prodotto in questione, destinato ad alimentazione per sonda (nutrizione enterale), è confezionato in sacche da 500 millilitri (packaging primario) che riportano le indicazioni su numero di sacca/lotto di produzione, fabbricante, scadenza e inoltre composizione, indicazioni cliniche, modo d'uso, avvertenze speciali e conservazione. Queste ultime indicazioni sono riportate in 11 lingue diverse, per evidenti esigenze di distribuzione in più $\mathrm{Pa}-$ esi europei, pertanto la superficie a disposizione è quasi totalmente coperta dalla stampa: un lato è completamente rivestito dalle scritte, l'altro ha una stretta colonna centrale trasparente per controllare il volume di prodotto residuo durante l'infusione.

Il colore utilizzato per lo sfondo (forse per esigenze estetiche, essendo l'alimento di colore nocciola), che sarebbe auspicabile per rendere più evidente il testo, impedisce invece la visione del prodotto. Le dimensioni del carattere sono piccole e la distribuzione del testo compressa per adattarsi alla superficie della sacca.

Di fatto quindi le notizie essenziali che l'operatore sanitario o il familiare/caregiver domiciliare dovrebbe immediatamente visualizzare risultano:

- difficili da trovare, in mezzo a tanta grafica e lingue diverse;

- difficili da leggere per la distribuzione del testo.

Nel caso descritto, quindi, l'impatto visivo con il prodotto contenuto nella sacca e le "Avvertenze speciali” («Non utilizzare se la sacca è gonfia o se il contenuto è coagulato») avrebbe causato un'erronea somministrazione, fortunatamente bloccata dal segnale della pompa.

\section{POMATA OMEOPATICA: NOME FUORVIANTE}

Il secondo caso riguarda una pomata omeopatica, utilizzata per la protezione del capezzolo da una mamma nei primi giorni di allattamento, che ha indotto nel neonato un disturbo gastrointestinale con diarrea, pianto e febbre. 


\section{CARTA DI RACCOMANDAZIONI PER IL "PACKAGING SICURO"}

\section{La forma del confezionamento}

Riguarda il senso della vista ma anche quello del tatto (es. stampa in carattere Braille per non vedenti). Lavorare sulla forma può fornire un elemento forte di riconoscibilità dei prodotti, ma si deve tener conto di molti fattori. Le regole basilari sono:

- usare confezioni differenziate (non una scatola dello stesso tipo in cui cambia solo il nome o il dosaggio);

- stampigliare le "informazioni critiche" (nome, dosaggio e forma farmaceutica) su almeno tre lati non opposti;

- orientare il testo nella stessa direzione;

- idem per i codici a lettura ottica, che devono inoltre avere adeguate dimensioni;

- se la confezione è colorata usare spazi bianchi per enfatizzare le informazioni critiche;

- creare continuità e corrispondenza tra gli stili grafici della confezione primaria e secondaria, ma anche tra queste e foglietti illustrativi o etichette.

\section{I materiali}

Nella scelta dei materiali non si deve optare per carte, cartone, pellicole o materiale riflettente come base per la stampa, sia sul confezionamento interno (es. blister) che esterno.

3. II carattere tipografico (tipo e font)

Si tratta di un elemento da non sottovalutare, che dovrebbe essere oggetto di studio: la scelta di un font piuttosto che un altro, della dimensione, della spaziatura tra le lettere e tra le righe, è fondamentale per determinare la leggibilità dei contenuti da parte degli utilizzatori. Questi possono essere anziani con capacità visive ridotte o persone con disturbi che ne alterano le facoltà percettive o operatori sanitari con necessità di operare in fretta $o$ in condizioni di superlavoro. La chiarezza della stampa è quindi imprescindibile per evitare errori e scambi di dosaggio o prodotto. $\mathrm{Di}$ fondamentale importanza è poi la creazione di una continuità grafica e di corrispondenza tra gli stili adottati per la confezione primaria e la secondaria, ma anche tra queste, le etichette e i foglietti illustrativi.

\section{La priorità delle informazioni}

La grafica deve evidenziare e scandire le informazioni più importanti, in modo che queste possano essere immediatamente visualizzate: nome del prodotto, principio attivo, dosaggio e forma farmaceutica. Per renderle facilmente riconoscibili nell'ambito di tutta la gestione del farmaco, sarebbe opportuno indicarle su tutte e sei le facce della confezione esterna (packaging secondario) e comunque non meno che su tre facce. Nell'ambito del confezionamento primario:

- le etichette di fiale o flaconi devono essere di dimensioni adeguate e riportare le indicazioni essenziali in modo chiaro e completo;

- il testo deve essere orientato tutto nella stessa direzione;

- i blister devono avere dimensioni tali da poter riportare sotto a ciascun alloggiamento del medicinale, il nome e il dosaggio;

- il foglio illustrativo deve essere impostato per far recepire rapidamente al primo esame le indicazioni su tipo di farmaco, dosaggio, indicazioni terapeutiche e controindicazioni (mediante un layout grafico facilmente leggibile).

5. II colore

In generale il colore è usato per differenziare dosaggi o specialità diverse della stessa azienda, ma il suo abuso può generare una visione distorta del prodotto. Per armonizzare cromatismi eccessivi, si potrebbe ipotizzare un utilizzo sistematico dei colori in modo da creare un codice cromatico internazionale di classificazione es. per gruppo ATC (classificazione anatomico terapeutica) dei farmaci. In alcuni ospedali, nelle sale operatorie viene già usato un codice colore per distinguere i farmaci. Nello studio del cromatismo non si deve tralasciare il contrasto testo-sfondo, che non deve pregiudicare la leggibilità, anzi deve esaltarla. Ad esempio se la confezione è colorata è utile usare spazi bianchi per enfatizzare le informazioni critiche.

\section{II nome commerciale}

Ogni azienda farmaceutica assegna alle specialità medicinali nomi di fantasia, che possono evocare il nome del principio attivo, della patologia cui sono destinati o l'azione che esplicano. Troppo spesso però si incontrano nomi simili per farmaci totalmente diversi, che possono generare scambi ed errori terapeutici, riscontrabili in numerosi esempi di letteratura. Si raccomanda quindi l'attenta analisi dei nomi commerciali esistenti, al momento della creazione di uno nuovo. Meglio se si potesse usufruire di un apposito database, in grado di fornire un controllo puntuale su ogni eventuale similitudine con farmaci già in commercio a livello mondiale.

\section{La data di scadenza}

La data di scadenza costituisce sempre un elemento critico poiché, per motivi pratici ed economici, viene stampigliata in un secondo tempo sul packaging primario e secondario, ad inchiostro o punzonata senza colore, e spesso con font grafico minuto. Ciò comporta inevitabilmente difficoltà di lettura e possibili errori sulla qualità del farmaco, nel senso dell'editabilità e della somministrazione. La data di scadenza deve essere facilmente visibile, per grafica e grandezza del carattere, sul confezionamento secondario e primario; in questo, se si tratta di blister o monodosi, la data dovrebbe essere stampata per ogni unità terapeutica. 
La pomata è un prodotto omeopatico derivato dall'olio di ricino, universalmente conosciuto per le proprietà purganti. Il nome riferito alla sostanza di base in inglese non evoca il ricino e la confezione non contiene foglio illustrativo come in altri prodotti omeopatici; le uniche indicazioni riguardano l'applicazione per uso locale.

Il prodotto, facilmente reperibile anche via internet a prezzi molto vantaggiosi, è descritto sul web da molte opinioni degli utilizzatori come «prodotto non tossico, miracoloso, che funziona alla grande». Non è quindi indicata da nessuna parte la possibilità di assorbimento nel lattante.

In questo caso si ha una duplicità di assorbimento e azione, esplicata dall'olio di ricino. L'uso locale della preparazione è corretto per le proprietà emollienti sulla mamma ma, anche se il capezzolo viene lavato prima dell'attaccamento al seno, l'assorbimento nel neonato può avvenire per uso sistemico attraverso il latte, prevalendo la proprietà lassativa e irritante del principio attivo.

A ciò si aggiunge la percezione di "sicurezza d'uso" derivante dalla natura stessa del prodotto (derivazione vegetale, quindi naturale e non dannoso) per mettere a rischio una popolazione particolarmente sensibile come quella dei neonati.

\section{CREMA ANTICELLULITE: AVVERTENZE NON RIPORTATE}

Una signora, affetta da aritmie che hanno richiesto l'impianto di un defibrillatore, decide di utilizzare una crema anticellulite. Dopo una decina di appli-

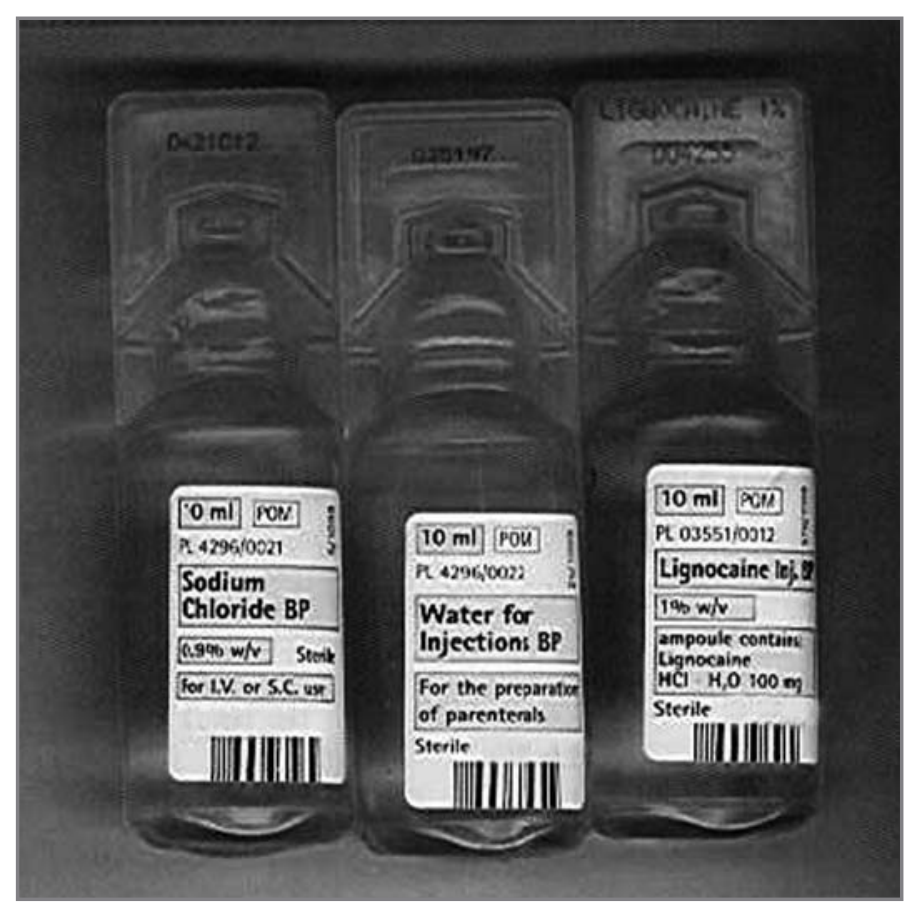

Figura 1. Esempio di packaging a scarso impatto visivo per differenziare farmaci e dosaggi diversi cazioni la signora viene ricoverata in terapia intensiva cardiologica in seguito a una tempesta aritmica, che ha determinato oltre 20 scariche al defibrillatore. La particolarità dell'evento, non spiegabile clinicamente in altro modo, porta lo specialista a indagare sulle altre possibili cause non strettamente legate alla patologia, individuando come unico evento concomitante l'impiego della crema anticellulite.

Anche in questo caso sul foglio illustrativo mancavano le avvertenze d'uso, in specie per pazienti già a rischio di eventi cardiovascolari, per derivazione dalle conoscenze su proprietà ed effetti dei componenti principali.

\section{PROBLEMATICHE E SCENARI}

Le normative nazionali e internazionali non impongono alle aziende farmaceutiche un confezionamento basato su precise indicazioni grafiche, ma solo obblighi su etichettatura e imballaggi (es. tappi del confezionamento primario, indicazioni essenziali per medicinali ad uso umano, omeopatici, medicinali in sperimentazione, ecc.).

Recentemente anche il Decreto Legislativo n. 219 del 24/4/2006 ("Codice comunitario dei medicinali"), che attua la Direttiva Europea 2001/83, definisce e specifica l'etichettatura e il foglietto illustrativo (art. 72 e seguenti), elenca la "tipologia" (art. 73) delle indicazioni da riportare sul confezionamento primario e/o secondario (denominazione, dosaggio e forma farmaceutica, composizione qualitativa e quantitativa, modalità e via di somministrazione, scadenza, lotto di produzione, numero AIC, prezzo); di fatto mancano però le indicazioni sulle specifiche grafiche, anche come indicazioni di minima, per prevenire gli errori di terapia.

Di "packaging sicuro" si discute a livello internazionale, si pubblicano brochure e articoli, si condividono raccomandazioni, ma manca ancora la fattiva collaborazione tra aziende farmaceutiche e professionisti esperti (Figura 1).

La situazione di rischio derivante dal packaging è complicata da un mercato farmaceutico in continua espansione, dal commercio su internet, dalla libera vendita dei farmaci per automedicazione, dalle importazioni parallele di prodotti industriali da Paesi membri dell'Unione Europea (dove il costo di produzione è più conveniente), dal gran numero di molecole che escono dalla brevettualità per trasformarsi in farmaci equivalenti e dal business che renderà appetibile questo mercato da parte di molti più produttori, anche neofiti o solo attirati da facili guadagni. E in assenza di specifiche normative, il packaging sarà probabilmente sacrificato a motivi di minor costo/massimo profitto. 
A tutto questo si aggiungano utilizzatori più disinvolti (che accedono a prodotti pericolosi anche solo da schermo e tastiera) e operatori sanitari stranieri, con scarsa padronanza della lingua italiana.

\section{GLI ASPETTI NORMATIVI}

\section{IL PACKAGING FARMACEUTICO: NON SOLO STILE}

Il packaging (ovvero l'imballaggio, la confezione o l'involucro) è diventato - non solo nel settore farmaceutico - uno strumento strategico per la promozione di qualsiasi prodotto.

Nel settore farmaceutico il produttore deve coniugare la perfezione tecnica dei contenitori e le esigenze funzionali del packaging con il rispetto delle regole di fabbricazione imposte per il settore.

Le norme di legge in tema di confezionamento dei prodotti farmaceutici ad uso umano sono poste a tutela della sanità pubblica e, precisamente, sono finalizzate a evitare che i consumatori siano indotti in errore.

La Direttiva 2001/83/CE ha istituto un codice comunitario relativo ai medicinali per uso umano ed è stata posta in essere proprio allo scopo di armonizzare la frammentaria disciplina della materia. La norma comunitaria è stata recepita nel nostro ordinamento dal Decreto Legislativo n. 219 del 24 aprile 2006 (poi integrato dal Decreto legislativo 29 dicembre 2007, n. 274 recante Disposizioni correttive al Decreto Legislativo n. 219 del 24/4/2006).

Secondo lo schema legislativo europeo, ogni Direttiva premette al testo della norma $\mathrm{i}$ cosiddetti considerando che individuano le ragioni che sono poste a base della norma promulgata e gli scopi che la norma stessa si prefigge di raggiungere.

Nei considerando della direttiva in analisi, con riferimento specifico al confezionamento dei prodotti medicinali, troviamo, infatti, che: «(2) Lo scopo principale delle norme relative alla produzione, alla distribuzione e all'uso di medicinali deve essere quello di assicurare la tutela della sanità pubblica. (3) Tuttavia questo scopo deve essere raggiunto avvalendosi di mezzi che non ostacolino lo sviluppo dell'industria farmaceutica e gli scambi dei medicinali nella Comunità. (4) Le disparità fra talune disposizioni nazionali e, in particolare, fra le disposizioni relative ai medicinali, eccettuate le sostanze o composizioni che sono derrate alimentari, alimenti destinati agli animali o prodotti d'igiene hanno per effetto di ostacolare gli scambi dei medicinali nella Comunità, e esse hanno, pertanto, un'incidenza diretta sul funzionamento del mercato interno. (5) Occorre, di conseguenza, eliminare questi ostacoli e per conseguire tale obiettivo si rende necessario un ravvicinamento delle suddette disposizioni. (6) Per ridurre le disparità che sussistono occorre stabilire le norme relative al controllo dei medicinali e inoltre precisare i compiti che spettano alle autorità competenti degli Stati membri per garantire il rispetto delle disposizioni di legge. [...]

(14) La presente direttiva costituisce una tappa importante nella realizzazione della libera circolazione dei medicinali; tuttavia tenuto conto dell'esperienza acquisita, in particolare in seno al comitato per le specialità medicinali, potrebbero rivelarsi necessarie nuove misure per eliminare gli ostacoli alla libera circolazione ancora esistenti. [...]

(21) In considerazione delle caratteristiche particolari dei medicinali omeopatici, quali il loro bassissimo tenore di principi attivi e la difficoltà di applicare loro la convenzionale metodologia statistica relativa alle prove cliniche, appare opportuno istituire una procedura specifica semplificata di registrazione per i medicinali omeopatici immessi in commercio senza particolari indicazioni terapeutiche ed in una forma farmaceutica ed un dosaggio che non presentino alcun rischio per il paziente. $[$...]

(23) È opportuno fornire innanzitutto ai consumatori di medicinali omeopatici un'indicazione molto chiara circa il carattere omeopatico degli stessi nonché sufficienti garanzie di qualità e di innocuità.

(24) È necessario armonizzare le norme riguardanti la fabbricazione, il controllo e l'ispezione dei medicinali omeopatici allo scopo di consentire la circolazione nell'intera Comunità di medicinali sicuri e di buona qualità. [...]

(39) È opportuno precisare le modalità secondo cui deve essere realizzata l'etichettatura e redatto il foglietto illustrativo.

(40) Le disposizioni relative alle informazioni da fornire ai pazienti devono garantire un livello elevato di tutela dei consumatori, così da permettere un impiego corretto dei medicinali sulla base di informazioni complete e comprensibili.

(41) L'immissione in commercio dei medicinali la cui etichettatura ed il cui foglietto illustrativo siano realizzati conformemente alla presente direttiva non deve essere vietata od impedita per motivi connessi all'etichettatura o al foglietto illustrativo. $[\ldots]$

(43) Tutti gli Stati membri hanno altresì adottato misure specifiche per la pubblicità per i medicinali; che tali misure presentano disparità che incidono sul funzionamento del mercato interno in quanto la pubblicità diffusa in uno Stato membro potrebbe avere effetti in altri Stati membri [...]». Quindi «(53) è opportuno che tutte le imprese produttrici o importatrici di medicinali istituiscano un sistema atto ad assicurare che le informazioni 
comunicate su un dato medicinale siano conformi alle condizioni di impiego approvate».

La norma comunitaria offre poi all'interprete le definizioni utili a interpretare univocamente la disposizione legislativa.

Con riferimento al confezionamento troviamo che per confezionamento primario si intende il contenitore o qualunque altra forma di confezionamento che si trova a diretto contatto con il medicinale. Per imballaggio esterno si intende l'imballaggio in cui è collocato il confezionamento primario. Per etichettatura si intendono le diciture iscritte sull'imballaggio esterno o sul confezionamento primario. Per foglietto illustrativo si intende il foglietto che reca informazioni destinate all'utente e che accompagna il medicinale.

La norma detta poi al titolo V rubricato "Etichettatura e foglietto illustrativo" una serie specifica di indicazione stabilendo, in particolare, all'art. 65 che: «all'occorrenza la Commissione pubblica linee direttrici riguardanti, in particolare quanto segue:

- la formulazione di alcune avvertenze speciali per determinate categorie di medicinali;

- le esigenze d'informazione particolari riguardanti l'autosomministrazione;

- la leggibilità delle indicazioni che figurano sull'etichettatura e sul foglietto illustrativo;

- l'impiego di codici per contraddistinguere ed autenticare i medicinali;

- l'elenco degli eccipienti che devono comparire nell'etichettatura dei medicinali e il modo di indicare detti eccipienti».

Dalla lettura della norma comunitaria emerge con evidenza un forte interesse della Comunità alla regolamentazione e all'uniformazione dell'etichettatura dei medicinali; tuttavia non si individuano prescrizioni specifiche che possano ovviare ai problemi diciamo "pratici" di lettura. La norma è, infatti, molto specifica sulle informazioni che devono essere fornite al consumatore, mentre non si occupa delle problematiche inerenti il packaging vero e proprio del prodotto, quali ad esempio l'uniformità del carattere dei foglietti illustrativi al fine di renderli chiari non solo nel contenuto ma anche nella struttura, avuto altresì riguardo al target del consumatore sul mercato.

Unica indicazione specifica presente nella norma si rinviene all'art. 69 in riferimento ai medicinali omeopatici, in relazione ai quali è stabilito che l'indicazione "medicinale omeopatico", deve essere posta in grande evidenza.

In relazione alla normativa vigente, la giurisprudenza si confronta con i problemi quotidiani. La Corte di giustizia, chiamata a classificare un prodotto (medicinale o non), effettua un'analisi della posizione del consumatore per individuare ciò che il medesimo pensa, o può essere indotto a pensare, allorché si accinge ad acquistare un prodotto. Emerge quindi la focalizzazione sull'interes- se del consumatore e sulla ricerca della maggiore chiarezza possibile a vantaggio del medesimo. Ad esempio si riporta di seguito ciò che la Corte ha espresso in merito a un preparato a base di aglio: «Non soddisfa i criteri di cui all'art. 1 , punto 2 della direttiva 2001/83 e non rientra nella definizione di medicinale per presentazione né in quella di medicinale per funzione, un preparato a base d'aglio, in capsule, che non è descritto né raccomandato come prodotto con capacità curative. In effetti, nessun aspetto relativo al suo confezionamento tende a far sì che tale prodotto assomigli ad un medicinale, se non la presenza, sulla confezione, della fotografia di una testa d'aglio. La presenza della fotografia di una pianta sulla confezione di un prodotto non può essere tuttavia sufficiente ad ispirare a un consumatore mediamente avveduto una fiducia del tipo di quella che ispirano normalmente i medicinali. Pertanto, la presentazione in forma di capsule è l'unico aspetto che può deporre in senso favorevole alla classificazione del prodotto quale medicinale per presentazione. In effetti, la forma esterna data ad un prodotto, pur rappresentando un indizio attendibile dell'intenzione del venditore o del fabbricante di metterlo in commercio come medicinale, non può costituire un indizio esclusivo e determinante, se non si vogliono comprendere taluni prodotti alimentari tradizionalmente presentati in forme analoghe a quelle dei medicinali. Tuttavia, la forma di capsula non è peculiare dei medicinali. Un gran numero di prodotti alimentari è infatti proposto in tale forma per rendere più agevole la loro assunzione da parte dei consumatori. Di conseguenza, tale indizio, da solo, non può essere sufficiente ad attribuire al prodotto controverso la qualità di medicinale per presentazione» (Corte giustizia CE, sez. I, 15 novembre 2007, n. 319, Comm. Ue c. Rep. Germania in Diritto \& Giustizia 2008).

\section{CONCLUSIONI}

In ospedale sistemi avanzati di gestione dei farmaci possono risolvere o annullare molti dei problemi creati da un packaging "non sicuro", ma il problema rimane per tutti i prodotti utilizzati a scopo medicamentoso e nell'ambito di tutto il ciclo produttivo, distributivo e gestionale: farmacie aperte al pubblico, parafarmacie, ambulatori, residenze per anziani o abitazioni.

Nella maggior parte dei casi le situazioni di rischio legate al confezionamento sono molte, perché non viene rispettato il principio User Centered Design, che pone l'utilizzatore/gestore finale (farmacista, infermiere, medico, paziente e cittadino in genere) al centro del processo di progettazione. 
Così, in un mercato farmaceutico in espansione, con prodotti e produttori di ogni tipo, con utilizzatori sempre più auto-internet-informati o non adeguatamente informati, con professionisti sanitari sempre più oberati e in presenza di operatori stranieri dalla formazione professionale e linguistica di vario livello, il packaging e la sua progettazione assumono un ruolo di assoluta importanza per la salute di tutti i cittadini.

Le Aziende che sapranno distinguersi in eccellenza per ricerca (su tecnologie, forme farmaceutiche, contenitori/dispositivi, innovazioni nel packaging) finalizzata alla riduzione di errori con attenzione agli utilizzatori (di tutta la filiera dalla produzione alla somministrazione) saranno quelle che vinceranno la sfida più ambiziosa del mercato globale farmaceutico: la sicurezza dei pazienti, primo obiettivo, peraltro, di ogni azienda farmaceutica.

Anche le Aziende che producono dispositivi, sistemi logistici e informatici dovranno adoperarsi affinché il Technology Assessment sia più Health Technology che solo Technology.

Servono infine le esperienze/conoscenze maturate da tutti i professionisti che si dedicano alla salute (dai designer ai clinici) per costruire un avanzamento a favore della comunità, indipendentemente dai singoli ruoli o interessi.

\section{BIBLIOGRAFIA DI RIFERIMENTO}

1. D. Lgs. 219/2006; Gazzetta Ufficiale n.142 del 21-06-2006, Supplemento Ordinario n.153

2. EIDD. Design for All Europe. The EIDD Stockholm Declaration. Stoccolma: 2004

3. Pazzagli L, Ranzani F. Packaging dei medicinali. Ergonomia al servizio della sicurezza dei pazienti e dei sistemi sanitari. Quaderni di Ergonomia 2006; 6: 11-6

4. Pazzagli L, Ranzani F. SIFO e Toscana lanciano il packaging a sicurezza garantita. Se farmacisti e designer si dessero la mano. Il Sole 24 ore Sanità 2006; 12: 23

\section{CORRESPONDING AUTHOR}

\section{Per la parte clinica:}

Dott.ssa Luciana Pazzagli, e-mail: luciana.pazzagli@asf.toscana.it

Dott.Francesco Ranzani, e-mail:francesco.ranzani@regione.toscana.it

Per la parte normativa:

Avv. Elena Albini, e-mail elena.albini@albini.eu, website: www.albini.eu 